Article

\title{
Weight Gain and Change in Body Mass Index after Age 20 in the Brazilian Population and Associated Sociodemographic Factors: Data from the National Health Survey
}

\author{
Nathalia A. B. Souza ${ }^{1}$, Karina A. Rimes-Dias ${ }^{1}$, Janaina C. Costa ${ }^{2}$ (D) and Daniela S. Canella ${ }^{3, *(D)}$ \\ 1 Postgraduate Program in Food, Nutrition and Health, Rio de Janeiro State University, \\ Rio de Janeiro 20550-013, Brazil; brigidonathalia@gmail.com (N.A.B.S.); \\ karinarimes@hotmail.com (K.A.R.-D.) \\ 2 International Center for Equity in Health, Federal University of Pelotas, Pelotas 96020-220, Brazil; \\ calu.janaina@gmail.com \\ 3 Department of Applied Nutrition, Institute of Nutrition, Rio de Janeiro State University, \\ Rio de Janeiro 20550-013, Brazil \\ * Correspondence: daniela.canella@uerj.br
}

Citation: Souza, N.A.B.; Rimes-Dias, K.A.; Costa, J.C.; Canella, D.S. Weight Gain and Change in Body Mass Index after Age 20 in the Brazilian Population and Associated Sociodemographic Factors: Data from the National Health Survey. Int. J. Environ. Res. Public Health 2022, 19, 2851. https://doi.org/10.3390/ ijerph19052851

Academic Editor: Juan Pablo

Rey-López

Received: 26 January 2022 Accepted: 23 February 2022 Published: 1 March 2022

Publisher's Note: MDPI stays neutral with regard to jurisdictional claims in published maps and institutional affiliations.

Copyright: () 2022 by the authors Licensee MDPI, Basel, Switzerland. This article is an open access article distributed under the terms and conditions of the Creative Commons Attribution (CC BY) license (https:// creativecommons.org/licenses/by/ $4.0 /)$.

\begin{abstract}
Obesity is considered one of the main contemporary public health problems. We aim to assess changes in body weight and nutritional status in adulthood and the associated sociodemographic variables. We use data from the 2013 National Health Survey $(n=21,743)$. Changes in weight and body mass index (BMI) were calculated based on mean difference between measurements at age 20 and data collected at the interview, stratified by sex. The association was analyzed using linear regression. Mean weight gain was greater among women than men. The largest gain was verified among the younger adults for both sexes. Age was found to be associated with weight and BMI change in men and women where, for every additional year of age, there was an increase in weight and BMI of $0.10 \mathrm{~kg}$ and $0.04 \mathrm{~kg} / \mathrm{m}^{2}$ in men and $0.22 \mathrm{~kg}$ and $0.09 \mathrm{~kg} / \mathrm{m}^{2}$ in women, respectively. For education, a direct association was found for men and an inverse for women. Association with area of residence was significant among males only, where rural men gained less than their urban counterparts. Weight gain was progressive, being more marked in the younger group, and was associated with education differently according to sex.
\end{abstract}

Keywords: obesity; excess weight; weight gain; health surveys; descriptive epidemiology

\section{Introduction}

Obesity is considered one of the main contemporary public health problems due to its high prevalence and the impact it produces both at individual and collective levels. Obesity is a multifactorial disease and one of its main causes is inadequate diet [1]. Between 1975 and 2016, the prevalence of obesity almost tripled worldwide, contributing to an increase in global deaths and to the emergence of non-communicable diseases (NCDs) attributable to excess adipose tissue. In 2017, excess weight (overweight and obesity) was estimated to have caused 2.4 million deaths and 70.7 million debilitating diseases in females, and 2.3 million deaths and 77.0 debilitating diseases in men $[2,3]$.

This rise in the prevalence of obesity can also be seen in Brazil. According to data from the Surveillance System of Risk and Protective Factors for Chronic Diseases by Telephone Survey (Sistema de Vigilância de Fatores de Risco e Proteção para Doenças Crônicas por Inquérito Telefônico-Vigitel), in 2006, a total of $11.4 \%$ of adults were obese [4]. Between 2010 and 2014, this rate rose from $15 \%$ to $18 \%$ in both sexes. In 2016 , an estimated $18.9 \%$ of Brazilian adults had obesity, with slightly higher rates observed among women (19.6\%) than men (18.1\%) [5]. In 2019, there was a trend of rising obesity rates, where the condition affected $20.3 \%$ of individuals, with similar rates in men and women [6]. 
Despite the fact that in the last survey the prevalence of obesity was similar between men and women, this was not the trend in recent decades. Theories proposed for this difference point to the effect of specific influences on weight gain of women, such as pregnancy and menopause [7]. Weight retention during pregnancy may also represent a determinant of obesity in women [8]. Furthermore, one of the major deleterious effects of declining estrogen is a change in women's body composition during menopause, altering the pattern of distribution of subcutaneous fat [9]. Besides biological factors, certain social aspects may also play a role in the greater weight gain among females. One hypothesis for this phenomenon is the difference in profession and family life dynamics for men and women. The pattern of inequality in the division of domestic tasks persists, even in developed countries, particularly when there are children, a spouse or an individual who needs special care. Thus, given the need to reconcile different demands, women may have less time for self-care, where this appears to have an adverse effect on health and on the capacity to maintain healthy life habits [10]. These aspects indicate the importance of investigating obesity by stratifying the analysis for sex.

A number of factors may have contributed to the occurrence of obesity, including poor diet, characterized by a switch from fresh or minimally processed food to ultra-processed foods [11-13]. Dietary patterns with a high intake of ultra-processed foods are typically rich in energy, free sugars, trans-fat and salt, and lacking in fiber and potassium. These characteristics can promote nutritional imbalances and impair the body's ability to control energy balance, increasing the risk of excess weight gain [14,15]. In addition to diet and sociodemographic factors, genetic factors can also have a role, even on a smaller scale, in the onset of obesity [16].

In this respect, excess weight gain in adulthood can result in increased nutritional risk, where individuals hitherto classified as normal weight start to become overweight or obese. Although in Brazil the trajectory of overweight and obesity is well documented, there is a dearth of reports on population-based studies assessing weight gain and nutritional status changes throughout the life span and exploring sex differences. Based on a representative sample of the Brazilian population, the objective of the present study was to assess changes in weight and nutritional status in adulthood and to identify the sociodemographic factors associated with this shift, examining their influence on men and women.

\section{Materials and Methods}

A cross-sectional study of data from the 2013 National Health Survey (Pesquisa Nacional de Saúde-PNS) was carried out. The PNS is a household survey conducted by the Brazilian Institute of Geography and Statistics (IBGE) in collaboration with the Oswaldo Cruz Foundation (FIOCRUZ) and the Ministry of Health [17].

The sampling plan employed by the PNS was a three-stage cluster sampling with geographic and socioeconomic stratification of primary sample units. Census sectors were the primary sampling units, households the second stage, and dwellers aged $>18$ years the third stage. Overall, a total of 81,254 households were visited, 69,994 of which were occupied. A total of 64,348 household interviews and 60,202 individual interviews with dwellers were carried out [17].

The main questions of interest in this study pertained to data on weight at 20 years of age and at the time of interview (present), the height of the individuals, and some sociodemographic characteristics. The data on weight at 20 years was collected based on the questions: "Do you remember what your approximate weight was at around 20 years of age?", "If yes, what was it?" and was applied only to respondents aged 30 or older. At the time of the interview, respondent weight and height were measured by trained researchers using a portable set of digital scales and a stadiometer.

Elderly people (>60 years old), pregnant women and individuals who did not answer the questions of interest were excluded from the analyses. Thus, the final sample comprised 21,743 adults, aged 30-59 years. 
Estimates of body mass index (BMI) at 20 years and BMI at time of interview were calculated by dividing body weight $(\mathrm{kg})$ by measured height squared (meters). Individuals were classified into: normal weight $\left(\mathrm{BMI}<25 \mathrm{~kg} / \mathrm{m}^{2}\right)$, overweight (BMI $\geq 25$ and $<30 \mathrm{~kg} / \mathrm{m}^{2}$ ) and obese (BMI $\geq 30 \mathrm{~kg} / \mathrm{m}^{2}$ ), at the two assessment time points, as per criteria defined by the WHO [18].

For the estimate of differences in weight and BMI, differences between current weight and weight at age 20 and between current BMI and BMI at age 20 were calculated. Shifts in the nutritional status classification of the participants between the age of 20 and current age were analyzed and expressed as a percentage of individuals belonging to each BMI category.

The relationship of sociodemographic characteristics with weight gain and BMI increase was explored. The sociodemographic variables evaluated were: race/skin color (white, black, mixed-race, Asian and indigenous), age (stratified into: 30-34, 35-39, 40-44, 45-50,51-54 and 55-59 years for the descriptive analyses, and used as a continuous variable in regression models), educational level (stratified into: 0-8, 9-11 and $\geq 12$ years of formal study), and area of residence (urban or rural). According to IBGE, urban areas correspond to cities (municipal seats), towns (district seats) or isolated urban areas, while rural areas covered the entire area located outside these limits.

Descriptive statistical analysis of all variables was performed to determine the percentage distribution of the characteristics of interest in the study population. Categorical variables were expressed as relative frequency and continuous variables as mean and respective $95 \%$ confidence intervals $(95 \% \mathrm{CI})$. All estimates of change in weight and BMI were calculated for Brazil and for each variable studied, stratified by sex. Significant differences were identified based on the comparison among the $95 \%$ CI. The absence of overlapping between intervals was assumed as a significant difference, considering the level of significance of $5 \%$. Lastly, crude and multiple linear regression models were employed to assess the association of sociodemographic variables with changes in weight and BMI. Variables that were significant on crude models (95\% CI did not include the null value) or that changed the adjusted coefficients were included in the multiple models. Adjustments were tested for each sex.

All data analyses were carried out using the statistics package Stata SE version 16.0 (Stata Corp., College Station, TX, USA), with the survey module considering the effects of complex sampling of the PNS, enabling extrapolation of the results for the whole adult Brazilian population.

\section{Results}

Of the 21,743 adults aged 30-59 years assessed, 55.1\% were women and $44.8 \%$ were men. The age distribution declined with increasing age. The sample was predominantly white, followed by black and mixed-race, while Asian and indigenous categories accounted for $1 \%$. The majority of individuals had 12 or more years of education. There were more dwellers from urban than rural areas (Table 1).

Weight and BMI distribution, at age 20 and at current age, according to sociodemographic characteristics and sex, are shown in Table 1 . At 20 years, mean weight and BMI of men were $66.1 \mathrm{~kg}(95 \%$ CI $65.7 ; 66.5)$ and $22.4 \mathrm{~kg} / \mathrm{m}^{2}(95 \%$ CI $22.3 ; 22.5)$, respectively, and at current age were $79.8 \mathrm{~kg}\left(95 \%\right.$ CI 79.2; 80.3) and $27.0 \mathrm{~kg} / \mathrm{m}^{2}$ (95\% CI 26.9; 27.2), representing a mean BMI increase of $20.7 \%$. For women, mean weight and BMI at 20 were $53.9 \mathrm{~kg}(95 \%$ CI $53.6 ; 54.2)$ and $21.3 \mathrm{~kg} / \mathrm{m}^{2}(95 \%$ CI $21.2 ; 21.4)$, and at current age were $69.6 \mathrm{~kg}(95 \%$ CI $69.2 ; 70.1)$ and $27.5 \mathrm{~kg} / \mathrm{m}^{2}$ (95\% CI 27.3; 27.7), representing a $29.1 \%$ increase in mean BMI. 
Table 1. Distribution of individuals for mean weight and body mass index (BMI), by sex. Brazil, 2013.

\begin{tabular}{|c|c|c|c|c|c|c|c|c|c|c|c|}
\hline \multirow{2}{*}{\multicolumn{2}{|c|}{$\begin{array}{l}\text { Sociodemographic } \\
\text { Variables }\end{array}$}} & \multicolumn{2}{|c|}{$\begin{array}{c}\text { Percentage in Sample } \\
\%(95 \% \text { CI })\end{array}$} & \multicolumn{2}{|c|}{$\begin{array}{c}\text { Weight at } 20 \text { Years } \\
\text { kg }(95 \% \mathrm{CI})\end{array}$} & \multicolumn{2}{|c|}{$\begin{array}{c}\text { Current Weight } \\
\text { kg }(95 \% \text { CI) }\end{array}$} & \multicolumn{2}{|c|}{$\begin{array}{l}\text { BMI at } 20 \text { Years } \\
\mathrm{kg} / \mathrm{m}^{2}(95 \% \mathrm{CI})\end{array}$} & \multicolumn{2}{|c|}{$\begin{array}{c}\text { Current BMI } \\
\mathrm{kg} / \mathrm{m}^{2}(95 \% \mathrm{CI})\end{array}$} \\
\hline & & Men & Women & Men & Women & Men & Women & Men & Women & Men & Women \\
\hline $\mathrm{Br}$ & & $44.8(44.1 ; 45.5)$ & $55.1(54.4 ; 55.8)$ & $66.1(65.7 ; 66.5)$ & $53.9(53.6 ; 54.2)$ & $79.8(79.2 ; 80.3)$ & $69.6(69.2 ; 70.1)$ & $22.4(22.3 ; 22.5)$ & $21.3(21.2 ; 21.4)$ & $27.0(26.9 ; 27.2)$ & $27.5(27.3 ; 27.7)$ \\
\hline \multirow{6}{*}{ Age group } & 30-34 years & $20.6(19.3 ; 21.8)$ & $21.3(20.3 ; 22.4)$ & $68.3(67.3 ; 69.2)$ & $55.4(54.9 ; 56.0)$ & $80.4(79.3 ; 81.5)$ & $67.6(66.8 ; 68.5)$ & $22.6(22.3 ; 22.9)$ & $21.4(21.9 ; 21.7)$ & $26.6(26.3 ; 27.0)$ & $26.2(25.8 ; 26.5)$ \\
\hline & $35-39$ years & $17.7(16.6 ; 18.9)$ & $18.8(17.8 ; 19.9)$ & $66.2(65.3 ; 67.1)$ & $55.3(54.5 ; 56.0)$ & $79.8(78.6 ; 81.1)$ & $70.5(69.3 ; 71.8)$ & $22.2(22.0 ; 22.5)$ & $21.6(21.3 ; 21.9)$ & $26.8(26.4 ; 27.1)$ & $27.6(27.1 ; 28.1)$ \\
\hline & 40-44 years & $15.0(13.9 ; 16.1)$ & $16.8(15.7 ; 17.9)$ & $66.3(65.5 ; 67.1)$ & $54.0(53.3 ; 54.6)$ & $80.8(79.5 ; 82.0)$ & $69.4(68.4 ; 70.4)$ & $22.4(22.2 ; 22.6)$ & $21.3(21.0 ; 21.5)$ & $27.3(26.9 ; 27.6)$ & $27.3(26.9 ; 27.7)$ \\
\hline & $45-50$ years & $16.6(15.4 ; 17.9)$ & $15.9(14.8 ; 17.1)$ & $65.3(64.3 ; 66.3)$ & $52.8(52.1 ; 53.6)$ & $79.4(78.0 ; 80.8)$ & $70.7(69.7 ; 71.8)$ & $22.3(22.0 ; 22.6)$ & $21.0(20.7 ; 21.3)$ & $27.1(26.7 ; 27.5)$ & $28.1(27.7 ; 28.5)$ \\
\hline & $51-54$ years & $15.8(14.5 ; 17.3)$ & $14.4(13.3 ; 15.5)$ & $64.7(63.7 ; 65.6)$ & $52.9(52.2 ; 53.7)$ & $78.6(77.2 ; 80.0)$ & $69.9(68.9 ; 71.0)$ & $22.3(22.0 ; 22.5)$ & $21.3(21.0 ; 21.6)$ & $27.0(26.6 ; 27.4)$ & $28.1(27.7 ; 28.5)$ \\
\hline & $55-59$ years & $14.0(12.8 ; 15.4)$ & $12.5(11.5 ; 13.5)$ & $64.9(63.3 ; 66.4)$ & $51.8(51.0 ; 52.6)$ & $79.5(77.7 ; 81.3)$ & $70.1(68.9 ; 71.4)$ & $22.6(22.0 ; 23.1)$ & $21.1(20.8 ; 21.4)$ & $27.6(27.6 ; 28.1)$ & $28.5(28.1 ; 29.0)$ \\
\hline \multirow{4}{*}{ Race/Skin color } & White & $51.1(49.5 ; 52.8)$ & $52.9(51.4 ; 54.4)$ & $67.3(66.7 ; 67.9)$ & $54.2(53.8 ; 54.6)$ & $81.7(80.9 ; 82.4)$ & $70.1(69.4 ; 70.7)$ & $22.5(22.3 ; 22.6)$ & $21.2(21.1 ; 21.4)$ & $27.3(27.1 ; 27.5)$ & $27.4(27.2 ; 27.7)$ \\
\hline & Mixed-race & $38.9(37.2 ; 40.5)$ & $37.1(35.7 ; 38.6)$ & $64.6(64.0 ; 65.1)$ & $53.3(52.8 ; 53.7)$ & $77.6(76.7 ; 78.5)$ & $68.5(67.9 ; 69.2)$ & $22.3(21.1 ; 22.4)$ & $21.4(21.1 ; 21.6)$ & $26.7(26.5 ; 27.0)$ & $27.5(27.2 ; 27.7)$ \\
\hline & Asian & $0.7(0.5 ; 1.0)$ & $1.1(0.8 ; 1.5)$ & $66.0(62.9 ; 69.2)$ & $52.5(50.7 ; 54.3)$ & $73.8(70.9 ; 76.6)$ & $64.6(60.7 ; 68.5)$ & $23.0(21.7 ; 24.2)$ & $21.4(20.7 ; 22.0)$ & $25.6(24.6 ; 26.7)$ & $26.2(24.8 ; 27.7)$ \\
\hline & Indigenous & $0.3(0.2 ; 0.5)$ & $0.5(0.3 ; 0.7)$ & $61.8(58.4 ; 65.1)$ & $52.2(49.8 ; 54.6)$ & $76.4(72.0 ; 80.9)$ & $68.3(62.5 ; 74.0)$ & $21.9(20.8 ; 23.0)$ & $21.6(20.5 ; 22.6)$ & $27.1(25.7 ; 28.5)$ & $28.2(25.9 ; 3.5)$ \\
\hline \multirow{3}{*}{ Educational level } & $0-8$ years & $34.5(32.6 ; 36.4)$ & $28.7(27.3 ; 30.3)$ & $63.6(62.9 ; 64.2)$ & $53.3(52.7 ; 53.9)$ & $76.0(75.0 ; 77.1)$ & $70.0(69.2 ; 70.8)$ & $22.2(21.9 ; 22.4)$ & $21.7(21.4 ; 21.9)$ & $26.4(26.1 ; 26.8)$ & $28.4(28.1 ; 28.8)$ \\
\hline & 9-11 years & $14.8(13.5 ; 16.3)$ & $14.5(13.5 ; 15.5)$ & $66.5(65.2 ; 67.8)$ & $53.3(52.6 ; 54.0)$ & $79.2(77.8 ; 80.6)$ & $70.1(69.1 ; 71.1)$ & $22.7(22.3 ; 23.1)$ & $21.3(21.0 ; 21.5)$ & $27.0(26.6 ; 27.5)$ & $28.0(27.6 ; 28.4)$ \\
\hline & $\geq 12$ years & $50.7(48.7 ; 52.7)$ & $56.8(55.1 ; 58.4)$ & $67.6(67.1 ; 68.2)$ & $54.4(54.0 ; 54.8)$ & $82.5(81.8 ; 83.2)$ & $69.3(68.7 ; 69.9)$ & $22.5(22.3 ; 22.6)$ & $21.1(21.0 ; 21.3)$ & $27.4(27.2 ; 27.6)$ & $26.9(26.7 ; 27.2)$ \\
\hline \multirow{2}{*}{ Area of residence } & Urban & $87.2(86.1 ; 88.1)$ & $90.5(89.7 ; 91.2)$ & $66.4(66.0 ; 66.9)$ & $54.0(53.7 ; 54.3)$ & $80.6(80.0 ; 81.2)$ & $69.7(69.2 ; 70.2)$ & $22.4(22.3 ; 22.6)$ & $21.3(21.2 ; 21.4)$ & $27.2(27.0 ; 27.4)$ & $27.5(27.3 ; 27.7)$ \\
\hline & Rural & $12.7(11.8 ; 13.8)$ & $9.4(8.7 ; 10.2)$ & $63.8(62.8 ; 64.7)$ & $53.6(52.7 ; 54.5)$ & $74.1(73.0 ; 75.1)$ & $69.0(67.8 ; 70.1)$ & $22.5(21.9 ; 22.5)$ & $21.5(21.2 ; 21.8)$ & $25.8(25.4 ; 26.1)$ & $27.7(27.2 ; 28.2)$ \\
\hline
\end{tabular}


A negative relation was observed between mean weight at age 20 and age at the time of interview in both sexes: individuals younger at the time of interview reported greater weight at 20 compared to those older at the time of interview. This same age effect was seen for current BMI in both sexes. With regard to race/skin color, there were differences for weight at 20 and current weight between white and black groups, although the difference for BMI was only observed among men, with higher estimates in the white population. For education, men with more years of education had a greater mean weight than those with fewer years of education, both at 20 years of age and current age, with the same pattern found for current BMI. By contrast, women with more years of education had lower current mean weight and BMI at 20 and current BMI compared to those with lower educational level. Additionally, mean weight at 20 years, current weight and current BMI were higher among urban males compared to their rural counterparts (Table 1).

The results for estimated weight gain and difference in BMI in adulthood are given in Table 2. Men gained an average of $13.7 \mathrm{~kg}$ (95\% CI 3.1; 14.2), or $4.6 \mathrm{~kg} / \mathrm{m}^{2}$ (95\% CI $\left.4.4 ; 4.7\right)$. The estimated weight gain and BMI change for the women were $15.6 \mathrm{~kg}(95 \%$ CI 15.2; 16.0) and $6.2 \mathrm{~kg} / \mathrm{m}^{2}(95 \% \mathrm{CI} 6.0 ; 6.3)$, respectively.

Table 2. Mean change in weight and BMI, by sex. Brazil, 2013.

\begin{tabular}{|c|c|c|c|c|c|}
\hline \multirow{2}{*}{\multicolumn{2}{|c|}{$\begin{array}{l}\text { Sociodemographic } \\
\text { Variables }\end{array}$}} & \multicolumn{2}{|c|}{$\begin{array}{c}\text { Difference in Weight } \\
\mathrm{kg}(95 \% \mathrm{CI})\end{array}$} & \multicolumn{2}{|c|}{$\begin{array}{l}\text { Difference in BMI } \\
\mathrm{kg} / \mathrm{m}^{2}(95 \% \mathrm{CI})\end{array}$} \\
\hline & & Men & Women & Men & Women \\
\hline \multicolumn{2}{|c|}{ Brazil } & $13.7(13.1 ; 14.2)$ & $15.6(15.2 ; 16.0)$ & $4.6(4.4 ; 4.7)$ & $6.2(6.0 ; 6.3)$ \\
\hline \multirow{6}{*}{ Age group } & 30-34 years & $12.1(11.2 ; 13.0)$ & $12.1(11.4 ; 12.9)$ & $4.0(3.7 ; 4.3)$ & $4.7(4.4 ; 5.0)$ \\
\hline & 35-39 years & $13.6(12.5 ; 14.6)$ & $15.2(14.2 ; 16.2)$ & $4.5(4.1 ; 4.8)$ & $5.9(5.5 ; 6.3)$ \\
\hline & 40-44 years & $14.4(13.3 ; 15.6)$ & $15.4(14.4 ; 16.3)$ & $4.8(4.4 ; 5.2)$ & $6.0(5.6 ; 6.4)$ \\
\hline & $45-50$ years & $14.0(12.8 ; 15.2)$ & $17.8(16.9 ; 18.8)$ & $4.7(4.3 ; 5.1)$ & $7.0(6.7 ; 7.4)$ \\
\hline & $51-54$ years & $13.9(12.5 ; 15.2)$ & $17.0(15.8 ; 18.1)$ & $4.7(4.3 ; 5.1)$ & $6.8(6.3 ; 7.2)$ \\
\hline & 55-59 years & $14.6(12.6 ; 16.5)$ & $18.3(17.0 ; 19.5)$ & $5.0(4.3 ; 5.6)$ & $7.4(6.9 ; 7.9)$ \\
\hline \multirow{5}{*}{ Race/skin color } & White & $14.3(13.7 ; 15.0)$ & $15.8(15.2 ; 16.3)$ & $4.7(4.5 ; 5.0)$ & $6.2(5.9 ; 6.4)$ \\
\hline & Black & $13.0(11.0 ; 15.1)$ & $17.1(15.5 ; 18.8)$ & $4.4(3.7 ; 5.1)$ & $6.7(6.0 ; 7.3)$ \\
\hline & Mixed-race & $13.0(12.2 ; 13.9)$ & $15.2(14.6 ; 15.8)$ & $4.4(4.1 ; 4.7)$ & $6.1(5.8 ; 6.3)$ \\
\hline & Asian & $7.7(3.1 ; 12.3)$ & $12.0(8.9 ; 15.2)$ & $2.6(1.0 ; 4.2)$ & $4.8(3.6 ; 6.1)$ \\
\hline & Indigenous & $14.6(11.1 ; 18.2)$ & $16.0(10.3 ; 21.8)$ & $5.1(3.8 ; 6.3)$ & $6.6(4.3 ; 8.9)$ \\
\hline \multirow{3}{*}{ Educational level } & $0-8$ years & $12.4(11.4 ; 13.4)$ & $16.7(15.9 ; 17.4)$ & $4.2(3.9 ; 4.6)$ & $6.7(6.4 ; 7.0)$ \\
\hline & $9-11$ years & $12.7(11.2 ; 14.1)$ & $16.8(15.7 ; 17.8)$ & $4.3(3.8 ; 4.8)$ & $6.7(6.2 ; 7.1)$ \\
\hline & $\geq 12$ years & $14.8(14.1 ; 15.5)$ & $14.8(14.3 ; 15.4)$ & $4.9(4.7 ; 5.1)$ & $5.7(5.5 ; 5.9)$ \\
\hline \multirow{2}{*}{ Area of residence } & Urban & $14.2(13.6 ; 14.7)$ & $15.7(15.2 ; 16.1)$ & $4.7(4.5 ; 4.9)$ & $6.2(6.0 ; 6.3)$ \\
\hline & Rural & $10.3(9.4 ; 11.1)$ & $15.3(14.2 ; 16.4)$ & $3.5(3.2 ; 3.8)$ & $6.1(5.6 ; 6.6)$ \\
\hline
\end{tabular}

A significant difference in the increase in weight and BMI was noted only among men aged 40-44 relative to those aged 30-34, and across all older relative to younger age brackets for women. From age 20 to 30-34 years, there was a weight gain of $12.1 \mathrm{~kg}$ for both sexes. Mean weight gain from age 20 to $55-59$ years was $14.6 \mathrm{~kg}$ in men and $18.3 \mathrm{~kg}$ in women. Adults younger at the time of interview (30-34 years) were heavier at 20 years of age than individuals older at interview (55-59 years). This difference among males averaged $3.4 \mathrm{~kg}$ versus $3.6 \mathrm{~kg}$ among females (Table 2).

Males with $\geq 12$ years of education had a higher mean difference in weight $(14.8 \mathrm{~kg}$; $95 \%$ CI $14.1 ; 15.5)$ relative to those with $0-8$ years of education. In women, the largest difference in weight was found among those with 9-11 years of education $(16.8 \mathrm{~kg} ; 95 \% \mathrm{CI}$ $15.7 ; 17.8)$ relative to women with $\geq 12$ years of education. The difference in BMI showed similar results in both sexes. For the area of residence, weight and BMI differences were greater among men from urban areas, with differences in the weight of $14.2 \mathrm{~kg}$ (95\% CI 13.6; 14.7), versus averages of $10.3 \mathrm{~kg}(95 \%$ CI 9.4;11.1). The BMI difference presented results in the same direction as the weight difference (Table 2). 
Changes in the nutritional status of males from the age of 20 years to the current age are shown in Table 3. Of the men who had normal weight at 20 years, $45.4 \%$ (95\% CI 43.3; 47.5) were overweight and $16.9 \%(95 \%$ CI $15.5 ; 18.4)$ had obesity in adulthood. Of the overweight individuals at 20 years, only $17.3 \%(95 \%$ CI $14.3 ; 20.7)$ lost weight to attain a BMI classified as normal, and $44.3 \%(95 \%$ CI $40.2 ; 48.6)$ went on to obesity. Of those who had obesity at 20 years, only $10.1 \%$ (95\% CI 5.3; 18.2) attained normal weight, while $63.3 \%$ (95\% CI 50.2; 74.6) remained obese. Thus, less than $40 \%$ of individuals of normal weight at 20 years maintained adequate nutritional status, whereas over $60 \%$ of individuals who had obesity as younger adults remained so in later life.

Comparing individuals aged 30-34 years and 55-59 years, in the older group a lower proportion of those of normal weight at 20 remained so in adulthood $(30.9 \% ; 95 \%$ CI 26.1 ; $36.0)$, while a greater proportion developed obesity later in adulthood $(21.1 \%$; $95 \%$ CI 16.6 ; 26.3). For education, a lower proportion of high-educated ( $\geq 12$ years) individuals retained normal weight into adulthood $(34.1 \% ; 95 \%$ CI $31.5 ; 36.8)$, compared with lower educated ( $\leq 8$ and 9-11 years) groups. No significant differences according to race/skin color were found. Results for the area of residence revealed that a higher rate of rural dwellers maintained normal weight status compared with urban dwellers (Table 3).

The data on changes in nutritional status among women are presented in Table 4. Of the normal-weight women at 20 years, 38.5\% (95\% CI 37.0; 40.1) remained of normal-weight, $37.1 \%(95 \%$ CI 35.7; 38.6) were overweight, and $24.2 \%(95 \%$ CI $22.8 ; 25.6)$ had obesity. Of those who had obesity at 20 years, only $10.9 \%(95 \%$ CI $6.8 ; 16.9)$ attained normal weight, while $61.4 \%$ (95\% CI 52.5; 69.7) remained obese.

Similarly to the men, comparing women aged 30-34 and 55-59 years, in the older group a lower proportion of those of normal weight at 20 remained so in adulthood $(28.5 \%$; $95 \%$ CI $24.6 ; 32.6)$, while a greater proportion developed obesity $(21.1 \%$; $95 \%$ CI $16.6 ; 26.3)$. Conversely for education, a greater proportion of the high-educated women remained of normal weight into adulthood $(43.6 \% ; 95 \%$ CI 43.6; 45.8) and a lower proportion developed obesity $(20.8 \%$; 95\% CI 19.1; 22.7), compared with low-educated groups $(43.6 \%$; 95\% CI 41.5; 45.8 and $29.1 \% ; 95 \%$ CI 26.5; 31.9, respectively). No significant differences for race/skin color or area of residence were found (Table 4).

The results for association of sociodemographic variables with change in weight and BMI by sex are given in Table 5. On the crude analyses, an association with age, education and area of residence was found in men and an association with age and education in women. After adjusting for significant variables on crude analysis, the same associations persisted, albeit weaker for some aspects.

On the adjusted models, age was associated with a change in weight and BMI in both sexes, exhibiting an increase of $0.10 \mathrm{~kg}(95 \%$ CI $0.04 ; 0.16)$ and $0.04 \mathrm{~kg} / \mathrm{m}^{2}(95 \%$ CI $0.02 ; 0.06)$ for every additional year of age in men, versus $0.22 \mathrm{~kg}(95 \%$ CI $0.17 ; 0.26)$ and $0.09 \mathrm{~kg} / \mathrm{m}^{2}(95 \%$ CI 0.07; 0.11) in women. Educational level was associated with an increase in weight, but not BMI, among men, where individuals with education $\geq 12$ years exhibited a significant increase compared to those with $0-8$ years of education. High-educated women ( $\geq 12$ years of education) exhibited a decrease in BMI relative to those with lower education (0-8 years). Area of residence (rural or urban) was significantly associated with a change in weight and BMI among men only, where rural-dwelling men gained less weight and BMI compared to urban men (Table 5). 
Table 3. Percentage change in nutritional status (at age 20 years versus current age) in men. Brazil, 2013.

\begin{tabular}{|c|c|c|c|c|c|c|c|c|c|c|}
\hline \multirow{3}{*}{\multicolumn{2}{|c|}{$\begin{array}{l}\text { Sociodemographic } \\
\text { Variables }\end{array}$}} & \multicolumn{3}{|c|}{$\begin{array}{c}\text { Normal Weight at Age } 20 \\
\left(\leq 24.9 \mathrm{~kg} / \mathrm{m}^{2}\right)\end{array}$} & \multicolumn{3}{|c|}{$\begin{array}{l}\text { Overweight at Age } 20 \\
\left(25.0-29.9 \mathrm{~kg} / \mathrm{m}^{2}\right)\end{array}$} & \multicolumn{3}{|c|}{$\begin{array}{c}\text { Obesity at Age } 20 \\
\left(\geq 30.0 \mathrm{~kg} / \mathrm{m}^{2}\right)\end{array}$} \\
\hline & & $\begin{array}{c}\text { Current } \\
\text { Normal Weight }\end{array}$ & $\begin{array}{c}\text { Current } \\
\text { Overweight }\end{array}$ & $\begin{array}{l}\text { Current } \\
\text { Obesity }\end{array}$ & $\begin{array}{c}\text { Current } \\
\text { Normal Weight }\end{array}$ & $\begin{array}{c}\text { Current } \\
\text { Overweight }\end{array}$ & $\begin{array}{l}\text { Current } \\
\text { Obesity }\end{array}$ & $\begin{array}{c}\text { Current } \\
\text { Normal Weight }\end{array}$ & $\begin{array}{c}\text { Current } \\
\text { Overweight }\end{array}$ & $\begin{array}{l}\text { Current } \\
\text { Obesity }\end{array}$ \\
\hline & & $\%(95 \%$ CI $)$ & $\%(95 \%$ CI $)$ & $\%(95 \% \mathrm{CI})$ & $\%(95 \%$ CI $)$ & $\%(95 \% \mathrm{CI})$ & $\%(95 \%$ CI $)$ & $\%(95 \%$ CI $)$ & $\%(95 \% \mathrm{CI})$ & $\%(95 \%$ CI $)$ \\
\hline \multicolumn{2}{|c|}{ Brazil } & $37.6(35.5 ; 39.6)$ & $45.4(43.3 ; 47.5)$ & $16.9(15.5 ; 18.4)$ & $17.3(14.3 ; 20.7)$ & $38.2(34.3 ; 42.4)$ & $44.3(40.2 ; 48.6)$ & $10.1(5.3 ; 18.2)$ & $26.5(17.1 ; 38.6)$ & $63.3(50.2 ; 74.6)$ \\
\hline \multirow{5}{*}{ Age group } & 30-34 years & $43.9(39.4 ; 48.4)$ & $44.5(39.9 ; 49.2)$ & $11.5(9.2 ; 14.3)$ & $12.2(6.7 ; 21.2)$ & $44.9(35.6 ; 54.7)$ & $42.7(33.7 ; 52.3)$ & $2.1(0.6 ; 6.6)$ & $27.0(14.2 ; 45.3)$ & $70.8(52.5 ; 84.1)$ \\
\hline & 35-39 years & $41.1(36.7 ; 45.5)$ & $44.0(39.6 ; 48.4)$ & $14.8(12.0 ; 18.1)$ & $13.3(8.7 ; 19.6)$ & $38.2(29.3 ; 47.9)$ & $48.4(39.5 ; 57.4)$ & $26.5(8.3 ; 58.8)$ & $16.7(6.9 ; 35.3)$ & $56.7(30.9 ; 79.2)$ \\
\hline & 40-44 years & $35.3(31.2 ; 39.7)$ & $44.8(40.4 ; 49.3)$ & $19.7(16.3 ; 23.5)$ & $14.6(9.4 ; 22.1)$ & $39.7(31.4 ; 48.7)$ & $45.5(36.4 ; 54.9)$ & $5.9(1.3 ; 22.2)$ & $52.0(22.9 ; 79.8)$ & $41.9(17.3 ; 71.2)$ \\
\hline & $45-50$ years & $36.9(32.2 ; 41.9)$ & $44.1(39.4 ; 48.9)$ & $18.8(15.2 ; 23.0)$ & $20.6(13.8 ; 29.6)$ & $32.4(23.6 ; 42.7)$ & $46.8(36.6 ; 57.4)$ & $18.1(6.9 ; 39.7)$ & $12.8(4.1 ; 33.3)$ & $69.0(45.0 ; 85.8)$ \\
\hline & 51-54 years & $34.0(28.8 ; 39.7)$ & $47.9(42.2 ; 53.6)$ & $17.9(14.2 ; 22.3)$ & $22.5(13.7 ; 34.6)$ & $39.5(29.1 ; 51.0)$ & $37.9(27.5 ; 49.4)$ & $2.5(0.5 ; 11.4)$ & $68.7(38.7 ; 88.4)$ & $28.6(10.3 ; 58.2)$ \\
\hline \multirow{5}{*}{ Race/skin color } & White & $34.3(31.5 ; 37.2)$ & $47.6(44.6 ; 50.7)$ & $18.0(15.8 ; 20.3)$ & $12.6(9.2 ; 17.1)$ & $39.3(33.4 ; 45.6)$ & $47.9(41.8 ; 54.1)$ & $12.7(5.4 ; 27.0)$ & $27.6(14.7 ; 45.8)$ & $59.6(42.3 ; 74.8)$ \\
\hline & Black & $42.5(35.8 ; 49.5)$ & $38.0(31.7 ; 44.8)$ & $19.3(15.2 ; 24.3)$ & $25.0(15.6 ; 37.4)$ & $22.8(13.9 ; 35.0)$ & $52.1(36.7 ; 67.1)$ & $0.4(0.0 ; 4.8)$ & $12.8(2.2 ; 48.3)$ & $86.7(51.0 ; 97.6)$ \\
\hline & Mixed-race & $43.3(27.5 ; 60.6)$ & $50.8(33.8 ; 67.5)$ & $5.8(1.9 ; 16.3)$ & $37.8(9.1 ; 78.6)$ & $49.6(15.2 ; 84.4)$ & $12.4(2.7 ; 41.8)$ & 0 & 100.0 & 0 \\
\hline & Asian & $40.6(37.7 ; 43.6)$ & $44.1(41.3 ; 47.0)$ & $15.1(13.3 ; 17.2)$ & $21.4(16.2 ; 27.6)$ & $39.6(33.8 ; 45.8)$ & $38.8(33.1 ; 44.8)$ & $11.0(4.8 ; 23.3)$ & $29.4(16.9 ; 46.0)$ & $59.5(42.9 ; 74.1)$ \\
\hline & Indigenous & $42.6(23.2 ; 64.6)$ & $39.6(20.8 ; 62.2)$ & $17.6(5.9 ; 41.9)$ & $6.3(1.8 ; 19.6)$ & $37.8(13.1 ; 71.0)$ & $55.7(25.7 ; 82.0)$ & 0 & 100.0 & 0 \\
\hline \multirow{3}{*}{ Educational level } & $0-8$ years & $41.5(38.1 ; 45.0)$ & $41.9(38.6 ; 45.3)$ & $16.4(13.5 ; 20.8)$ & $25.7(12.7 ; 32.7)$ & $39.1(32.3 ; 46.4)$ & $35.0(28.1 ; 42.6)$ & $26.7(12.4 ; 48.3)$ & $28.7(15.3 ; 47.1)$ & $44.5(25.3 ; 65.5)$ \\
\hline & 9-11 years & $40.0(38.1 ; 45.0)$ & $43.0(38.0 ; 48.1)$ & $16.9(13.5 ; 20.8)$ & $19.8(12.2 ; 30.4)$ & $35.0(24.9 ; 46.7)$ & $45.1(33.8 ; 56.9)$ & $7.6(1.5 ; 30.8)$ & $35.7(10.1 ; 73.2)$ & $56.6(20.2 ; 87.0)$ \\
\hline & $\geq 12$ years & $34.1(31.5 ; 36.8)$ & $48.6(45.7 ; 51.4)$ & $17.2(15.3 ; 19.4)$ & $11.7(8.3 ; 16.1)$ & $38.5(33.2 ; 44.1)$ & $49.6(44.0 ; 55.3)$ & $2.8(1.3 ; 5.9)$ & $20.9(11.7 ; 34.5)$ & $76.1(62.7 ; 85.8)$ \\
\hline \multirow{2}{*}{ Area of residence } & Urban & $35.8(33.5 ; 38.1)$ & $46.3(44.0 ; 48.6)$ & $17.8(16.2 ; 19.5)$ & $16.6(13.4 ; 20.2)$ & $37.5(33.3 ; 41.9)$ & $45.8(41.3 ; 50.3)$ & $9.0(4.3 ; 17.8)$ & $26.7(16.7 ; 39.8)$ & $64.2(50.3 ; 76.1)$ \\
\hline & Rural & $49.1(44.9 ; 53.3)$ & $39.9(35.9 ; 44.0)$ & $10.9(8.9 ; 13.3)$ & $24.0(16.7 ; 33.2)$ & $45.2(34.9 ; 55.9)$ & $30.6(22.3 ; 40.5)$ & $21.4(6.6 ; 51.3)$ & $24.7(7.9 ; 55.5)$ & $53.8(21.7 ; 82.9)$ \\
\hline
\end{tabular}

Table 4. Percentage change in nutritional status (at age 20 versus current age) in women. Brazil, 2013.

\begin{tabular}{|c|c|c|c|c|c|c|c|c|c|c|}
\hline \multirow{3}{*}{\multicolumn{2}{|c|}{$\begin{array}{l}\text { Sociodemographic } \\
\text { Variables }\end{array}$}} & \multicolumn{3}{|c|}{$\begin{array}{c}\text { Normal Weight at Age } 20 \\
\left(\leq 24.9 \mathrm{~kg} / \mathrm{m}^{2}\right)\end{array}$} & \multicolumn{3}{|c|}{$\begin{array}{c}\text { Overweight at Age } 20 \\
\left(25.0-29.9 \mathrm{~kg} / \mathrm{m}^{2}\right)\end{array}$} & \multicolumn{3}{|c|}{$\begin{array}{c}\text { Obesity at Age } 20 \\
\left(\geq 30.0 \mathrm{~kg} / \mathrm{m}^{2}\right)\end{array}$} \\
\hline & & $\begin{array}{c}\text { Current } \\
\text { Normal Weight }\end{array}$ & $\begin{array}{c}\text { Current } \\
\text { Overweight }\end{array}$ & $\begin{array}{l}\text { Current } \\
\text { Obesity }\end{array}$ & $\begin{array}{c}\text { Current } \\
\text { Normal Weight }\end{array}$ & $\begin{array}{c}\text { Current } \\
\text { Overweight }\end{array}$ & $\begin{array}{l}\text { Current } \\
\text { Obesity }\end{array}$ & $\begin{array}{c}\text { Current } \\
\text { Normal Weight }\end{array}$ & $\begin{array}{c}\text { Current } \\
\text { Overweight }\end{array}$ & $\begin{array}{l}\text { Current } \\
\text { Obesity }\end{array}$ \\
\hline & & $\%(95 \% \mathrm{CI})$ & $\%(95 \% \mathrm{CI})$ & $\%(95 \% \mathrm{CI})$ & $\%(95 \% \mathrm{CI})$ & $\%(95 \% \mathrm{CI})$ & $\%(95 \% \mathrm{CI})$ & $\%(95 \% \mathrm{CI})$ & $\%(95 \% \mathrm{CI})$ & $\%(95 \% \mathrm{CI})$ \\
\hline \multicolumn{2}{|c|}{ Brazil } & $38.5(37.0 ; 40.1)$ & $37.1(35.7 ; 38.6)$ & $24.2(22.8 ; 25.6)$ & $16.4(13.1 ; 20.2)$ & $24.6(20.4 ; 29.2)$ & $58.9(54.0 ; 63.7)$ & $10.9(6.8 ; 16.9)$ & $27.5(20.6 ; 35.8)$ & $61.4(52.5 ; 69.7)$ \\
\hline \multirow{5}{*}{ Age group } & 30-34 years & $53.7(50.4 ; 56.9)$ & $32.5(29.4 ; 35.8)$ & $13.7(11.4 ; 16.3)$ & $15.4(9.0 ; 25.2)$ & $22.6(15.0 ; 32.6)$ & $61.8(51.3 ; 71.4)$ & $7.3(2.8 ; 17.7)$ & $38.9(22.9 ; 57.7)$ & $53.7(36.1 ; 70.4)$ \\
\hline & 35-39 years & $41.2(37.8 ; 44.6)$ & $36.5(33.2 ; 39.8)$ & $22.2(19.3 ; 25.4)$ & $12.8(8.2 ; 19.3)$ & $23.1(15.6 ; 32.8)$ & $63.9(52.9 ; 73.6)$ & $9.6(3.1 ; 26.0)$ & $23.1(12.4 ; 39.0)$ & $67.1(49.8 ; 80.7)$ \\
\hline & 40-44 years & $38.2(34.6 ; 41.9)$ & $36.7(33.3 ; 40.2)$ & $25.0(21.8 ; 28.5)$ & $22.3(13.6 ; 34.4)$ & $25.3(17.6 ; 35.0)$ & $52.2(41.0 ; 63.3)$ & $12.7(4.0 ; 33.5)$ & $24.5(10.4 ; 47.4)$ & $62.6(40.2 ; 80.7)$ \\
\hline & $45-50$ years & $31.8(28.0 ; 35.8)$ & $41.2(37.3 ; 45.2)$ & $26.8(23.2 ; 30.7)$ & $13.8(8.4 ; 21.8)$ & $19.3(10.9 ; 31.9)$ & $66.7(54.5 ; 77.1)$ & $13.8(4.2 ; 36.7)$ & $22.5(9.0 ; 46.0)$ & $63.5(37.3 ; 83.6)$ \\
\hline & 51-54 years & $30.0(26.2 ; 34.1)$ & $41.2(36.9 ; 45.6)$ & $28.7(24.9 ; 32.7)$ & $20.3(11.1 ; 34.1)$ & $27.4(15.3 ; 44.1)$ & $52.2(37.4 ; 66.6)$ & $11.9(3.7 ; 32.3)$ & $12.8(3.2 ; 39.3)$ & $75.1(49.0 ; 90.5)$ \\
\hline
\end{tabular}


Table 4. Cont.

\begin{tabular}{|c|c|c|c|c|c|c|c|c|c|c|}
\hline \multirow{2}{*}{\multicolumn{2}{|c|}{$\begin{array}{l}\text { Sociodemographic } \\
\text { Variables }\end{array}$}} & \multicolumn{3}{|c|}{$\begin{array}{c}\text { Normal Weight at Age } 20 \\
\left(\leq 24.9 \mathrm{~kg} / \mathrm{m}^{2}\right)\end{array}$} & \multicolumn{3}{|c|}{$\begin{array}{l}\text { Overweight at Age } 20 \\
\left(25.0-29.9 \mathrm{~kg} / \mathrm{m}^{2}\right)\end{array}$} & \multicolumn{3}{|c|}{$\begin{array}{c}\text { Obesity at Age } 20 \\
\left(\geq 30.0 \mathrm{~kg} / \mathrm{m}^{2}\right)\end{array}$} \\
\hline & & $\begin{array}{c}\begin{array}{c}\text { Current } \\
\text { Normal Weight }\end{array} \\
\%(95 \% \mathrm{CI}) \\
\end{array}$ & $\begin{array}{c}\begin{array}{c}\text { Current } \\
\text { Overweight }\end{array} \\
\%(95 \% \text { CI }) \\
\end{array}$ & $\begin{array}{c}\begin{array}{c}\text { Current } \\
\text { Obesity }\end{array} \\
\%(95 \% \mathrm{CI})\end{array}$ & $\begin{array}{c}\begin{array}{c}\text { Current } \\
\text { Normal Weight }\end{array} \\
\%(95 \% \mathrm{CI})\end{array}$ & $\begin{array}{c}\begin{array}{c}\text { Current } \\
\text { Overweight }\end{array} \\
\%(95 \% \text { CI })\end{array}$ & $\begin{array}{c}\begin{array}{c}\text { Current } \\
\text { Obesity }\end{array} \\
\%(95 \% \mathrm{CI})\end{array}$ & $\begin{array}{c}\begin{array}{c}\text { Current } \\
\text { Normal Weight }\end{array} \\
\%(95 \% \text { CI })\end{array}$ & $\begin{array}{c}\begin{array}{c}\text { Current } \\
\text { Overweight }\end{array} \\
\%(95 \% \mathrm{CI})\end{array}$ & $\begin{array}{c}\begin{array}{c}\text { Current } \\
\text { Obesity }\end{array} \\
\%(95 \% \mathrm{CI})\end{array}$ \\
\hline \multirow{5}{*}{ Race/skin color } & White & $39.7(37.6 ; 41.9)$ & $36.2(34.0 ; 38.4)$ & $23.9(21.9 ; 26.0)$ & $13.6(10.1 ; 18.1)$ & $27.5(21.5 ; 34.6)$ & $58.7(51.7 ; 65.4)$ & $14.6(7.6 ; 26.2)$ & $30.1(19.9 \cdot 42.8)$ & $55.1(41.7 ; 67.8)$ \\
\hline & Black & $34.5(29.4 ; 39.9)$ & $35.0(30.2 ; 40.2)$ & $30.3(25.4 ; 35.7)$ & $15.8(6.4 ; 33.9)$ & $16.7(9.1 ; 28.7)$ & $67.3(50.3 ; 80.7)$ & $17.2(4.3 ; 48.8)$ & $19.3(6.3 ; 45.8)$ & $63.3(37.6 ; 83.1)$ \\
\hline & Mixed-race & $52.9(37.3 ; 67.8)$ & $33.4(20.5 ; 49.3)$ & $13.6(6.0 ; 27.8)$ & $31.0(9.7 ; 65.1)$ & $2.6(0.3 ; 18.1)$ & $66.3(32.8 ; 88.7)$ & 0 & 0 & 100.0 \\
\hline & Asian & $37.1(34.7 ; 39.6)$ & $39.2(36.8 ; 41.7)$ & $23.5(21.5 ; 25.6)$ & $19.5(14.1 ; 26.4)$ & $23.8(17.4 ; 31.6)$ & $56.6(48.6 ; 64.2)$ & $6.5(3.2 ; 12.9)$ & $26.1(16.0 ; 39.5)$ & $67.2(53.5 ; 78.5)$ \\
\hline & Indigenous & $45.2(27.1 ; 64.6)$ & $26.9(15.2 ; 43.0)$ & $27.8(14.7 ; 46.4)$ & $0.2(0.0 ; 2.7)$ & $1.7(0.2 ; 10.1)$ & $97.9(89.6 ; 99.6)$ & 0 & $98.6(88.6 ; 99.8)$ & $13.7(0.1 ; 11.3)$ \\
\hline \multirow{2}{*}{ Educational level } & $0-8$ years & $31.0(28.4 ; 33.7)$ & $39.7(36.8 ; 42.8)$ & $29.1(26.5 ; 31.9)$ & $19.2(13.7 ; 26.2)$ & $25.5(18.4 ; 34.2)$ & $55.1(47.2 ; 62.8)$ & $15.2(7.9 ; 27.2)$ & $22.2(12.7 ; 35.8)$ & $62.5(47.5 ; 75.4)$ \\
\hline & $\geq 12$ years & $43.6(41.5 ; 45.8)$ & $35.4(33.5 ; 37.4)$ & $20.8(19.1 ; 22.7)$ & $14.6(10.4 ; 20.2)$ & $24.5(19.3 ; 30.0)$ & $60.7(53.7 ; 67.2)$ & $3.4(1.4 ; 7.7)$ & $35.5(24.7 ; 47.9)$ & $61.0(48.6 ; 72.0)$ \\
\hline \multirow{2}{*}{ Area of residence } & Urban & $38.9(37.3 ; 40.6)$ & $36.9(35.3 ; 38.5)$ & $24.1(22.6 ; 25.6)$ & $16.3(12.8 ; 20.5)$ & $22.8(18.8 ; 27.4)$ & $60.7(55.6 ; 65.6)$ & $11.3(6.9 ; 18.0)$ & $28.3(20.8 ; 37.3)$ & $60.2(50.6 ; 69.1)$ \\
\hline & Rural & $34.9(30.8 ; 39.2)$ & $39.6(35.7 ; 43.6)$ & $25.4(21.7 ; 29.4)$ & $17.1(10.2 ; 27.3)$ & $38.8(24.0 ; 56.0)$ & $43.9(30.2 ; 58.5)$ & $6.7(1.9 ; 20.4)$ & $19.8(7.3 ; 43.6)$ & $73.4(50.1 ; 88.3)$ \\
\hline
\end{tabular}

Table 5. Association of sociodemographic variables with weight and BMI increase, by sex. Brazil, 2013.

\begin{tabular}{|c|c|c|c|c|c|c|c|c|c|}
\hline \multirow{3}{*}{\multicolumn{2}{|c|}{$\begin{array}{l}\text { Sociodemographic } \\
\text { Variables }\end{array}$}} & \multicolumn{4}{|c|}{ Weight Increase } & \multicolumn{4}{|c|}{ BMI Increase } \\
\hline & & \multicolumn{2}{|c|}{ Crude Coefficient $(95 \% \mathrm{CI})$} & \multicolumn{2}{|c|}{ Adjusted Coefficient $(95 \% \mathrm{CI})$} & \multicolumn{2}{|c|}{ Crude Coefficient $(95 \%$ CI) } & \multicolumn{2}{|c|}{ Adjusted Coefficient $(95 \% \mathrm{CI})$} \\
\hline & & Men & Women & Men * & Women ** & Men & Women & Men * & Women ** \\
\hline \multicolumn{2}{|c|}{ Age in full years } & $0.07(0.01 ; 0.13)$ & $0.22(0.18 ; 0.27)$ & $0.10(0.04 ; 0.16)$ & $0.22(0.17 ; 0.26)$ & $0.03(0.01 ; 0.05)$ & $0.10(0.08 ; 0.12)$ & $0.04(0.02 ; 0.06)$ & $0.09(0.07 ; 0.11)$ \\
\hline Educational level & $\begin{array}{l}0-8 \text { years } \\
9-11 \text { years } \\
\geq 12 \text { years }\end{array}$ & $\begin{array}{c}\text { Reference } \\
0.24(-1.52 ; 2.02) \\
2.39(1.17 ; 3.61)\end{array}$ & $\begin{array}{c}\text { Reference } \\
0.11(-1.21 ; 1.44) \\
-1.80(-2.73 ;-0.93)\end{array}$ & $\begin{array}{c}\text { Reference } \\
0.02(-1.83 ; 1.79) \\
2.12(0.82 ; 3.41)\end{array}$ & $\begin{array}{c}\text { Reference } \\
0.59(-0.73 ; 1.93) \\
-1.04(-1.95 ;-1.38)\end{array}$ & $\begin{array}{c}\text { Reference } \\
0.03(-0.55 ; 0.63) \\
0.64(-0.23 ; 1.05)\end{array}$ & $\begin{array}{c}\text { Reference } \\
-0.03(-0.57 ; 0.50) \\
-0.98(-1.33 ;-0.62)\end{array}$ & $\begin{array}{c}\text { Reference } \\
-0.03(-0.64 ; 0.56) \\
0.58(0.15 ; 1.01)\end{array}$ & $\begin{array}{c}\text { Reference } \\
0.17(-0.36 ; 0.71) \\
-0.63(-0.99 ;-0.27)\end{array}$ \\
\hline Area of residence & $\begin{array}{l}\text { Urban } \\
\text { Rural }\end{array}$ & $\begin{array}{c}\text { Reference } \\
-3.89(-4.94 ;-2.85)\end{array}$ & $\begin{array}{c}\text { Reference } \\
-0.38(-1.60 ; 0.83)\end{array}$ & $\begin{array}{c}\text { Reference } \\
-3.13(-4.3 ;-1.94)\end{array}$ & & $\begin{array}{c}\text { Reference } \\
-1.21(-1.57 ;-0.85)\end{array}$ & $\begin{array}{c}\text { Reference } \\
-0.04(-0.55 ; 0.45)\end{array}$ & $\begin{array}{c}\text { Reference } \\
-1.02(-1.42 ;-0.61)\end{array}$ & \\
\hline
\end{tabular}




\section{Discussion}

Based on an analysis of representative data for the Brazilian adult population, less than $40 \%$ of individuals of normal weight at age 20 remained so after the age of 30, while over $60 \%$ of adults who had obesity at age 20 remained so into later life. These data highlight that the process of weight gain continued during adulthood. The data also showed different patterns of weight gain and changes in nutritional status between men and women according to socioeconomic characteristics.

Mean weight gain was greater among women than men, differing by approximately $2 \mathrm{~kg}$. This result of greater weight gain among women is consistent with findings of other studies [5,19-21]. In this respect, the relationship between sex and weight gain is complex, involving social and biological issues, where no sole cause can be identified or degree of impact of these aspects established. However, stratified analyses exploring differences between men and women can promote investigations that are more in-depth or involve other fields of knowledge, in a bid to further understand the role of gender in this outcome.

Data from the Vigitel 2019 for the 26 major Brazilian cities and the Federal District show that the prevalence of adult obesity was $20.3 \%$, with similar rates for men and women [6]. This statistic may indicate a shift in the pattern of weight gain in the Brazilian population with respect to sex, at least in the setting of major metropoles.

The obesity transition can be classified into four. Stage 1 is characterized by a higher prevalence of obesity in women with lower education at a lower economic level than those with a lower socioeconomic level. In stage 2 there is a large increase in the prevalence of obesity among adults, a smaller one among children, and an increase in the distance between the sexes and an increase in socioeconomic differences among women. At stage 3, a prevalence of obesity between those with lower socioeconomic status and those with higher socioeconomic status, and a platform in the prevalence, can be observed in women of high socioeconomic status and children. The putative final stage (stage 4) of the obesity transition would be a declining prevalence [22]. Considering the data analyzed in the present study, it can be considered that, in 2013, Brazil was in stage 1 of the obesity transition with a higher percentage of weight gain for females when compared to males.

Besides the difference in weight gain between sexes, differences across age groups were also evident. Among the Brazilian population studied, weight gain was not only found to be continuous, but the greater increase in body mass appeared to occur in the first decade of adulthood. Nonetheless, it is not possible to affirm that weight gain did not also occur at 30-34 years in older age groups, due to the cross-sectional character of the study. Moreover, the potential influence of the generational aspect between the age groups, a cohort effect, which might impact weight at 20 years, should also be taken into account. However, different national and international studies also pointed out that weight gain seems to be greater in the first decade of adult life [23-26].

Analyzing data from some major cities of Brazil, studies concluded that most individuals experience weight gain after age 20. In a sample of 875 individuals (30-59 years) from Goiânia city, the Goiás state capital, 63.6\% exhibited weight gain of over 10\% [23]. Another study, of 1341 adults (aged 21-30, 31-40, 41-50 and $\geq 51$ years) from the city of Florianópolis, the Santa Catarina state capital, found that most men and women showed an increase of over $10 \%$ in BMI, while a quarter of women and $14 \%$ of men showed a BMI increase of over 30\% [24]. In addition, a study based on seven cross-sectional surveys of Vigitel data, involving all major Brazilian cities, analyzed time trends for weight gain and change in nutritional status among men and women. In 2006, the greatest change in mean weight gain among males was observed in the 30-34 age bracket. By comparison, in 2012, the highest weight gain for men occurred in the 21-24 age bracket. For women, in 2006, the greatest increases in weight gain were found between $30-34$ and 35-44 years (3.7 kg) and for the 35-44 and 45-59 (4.2 kg) age groups. In 2012, however, the highest increase in body weight occurred at 21-24 (3.4 kg) and between 21-24 and 25-29 years (5.1 kg) [25].

Similar results were reported for other populations. A North-American study of over 1800 individuals aged 20-39 years assessed the nutritional status by age group. The study 
found an obesity rate of $25.7 \%$ in the $20-24.9$ years age group, $24.3 \%$ in the $25-29.9,24 \%$ in the 30-34.9, and $26.0 \%$ in the 35-39.9 years age group [26]. Given that weight gain increases during the lifespan, the rate of obesity is expected to be higher among older individuals, a pattern also seen in Brazil. Data from the Vigitel system showed higher rates of obesity in older age groups [6]. In Australia, a cohort study found that mean change in BMI for both males and females was positively associated with the year of birth, where the prevalence of excess weight showed greater increases in cohorts born more recently than in older cohorts [27]. This cohort effect might be explained by dietary intake, given that younger individuals typically have a higher consumption of ultra-processed foods than older individuals [28].

The transition between adolescence and early adulthood is a key risk period for developing obesity in both sexes and different ethnic groups [18]. This might be explained by the fact the transition marks a period of major behavioral, social and economic complexity, in which different degrees of autonomy and responsibility manifest. Entry into the job market and leaving the parental home are examples of the responsibility acquired by these young adults $[18,19]$. At this stage in life, the young individual becomes responsible for their diet. The higher consumption of ultra-processed foods, which are quickly prepared and highly palatable, may explain the weight gain in this population [20], together with the lower level of physical activity, due to less time available for leisure. Engagement in physical activity has declined over the years in both sexes [21]. A cohort study involving North Americans found that weight gain during adulthood was associated with a significantly greater risk of developing chronic diseases (type 2 diabetes, hypertension and cancers associated to obesity) and a lesser probability of healthy aging [22].

The results in the present study failed to find any association between race/skin color and weight gain, which could be due to a lack of precision in the estimative, considering the small sample of some groups. However, in Brazil, the prevalence of obesity is known to be greater among black women than white women [29]. The higher prevalence of NCDs among black and mixed-race people indicates that risk factors must be distributed differently according to race/skin color and sex [30]. Although the black and mixedrace population, particularly female, is more likely to belong to the low socioeconomic strata, institutional racism is co-responsible for inequalities in the provision of care. This situation, among other factors, limits the action of professionals in the field of diagnosis and treatment, and also in the delivery of care for other health-related conditions [31-33]. Black women have a higher risk of death because racial and gender inequalities combine. These aspects are viewed as social and cultural constructions which define male and female roles, establishing hierarchies in which men are considered superior to women [30]. Furthermore, considering race/skin color as a social variable and not a biological one, the educational level could be better for adjusting weight gain in the PNS sample. Therefore, the relationship between race/skin color and nutritional status warrants further investigation in future studies.

In the present study, educational level was considered a proxy of income [34]. Women who had studied for 12 years or more showed a negative association with change in weight and BMI, i.e., women with a higher educational level gained less weight than women with fewer years of formal study, while the opposite held true for men. The association of nutritional status with education and income was found in a study exploring social inequity and malnutrition in all its forms (undernutrition and obesity) in the Brazilian population. Income and education exhibited similar associations to those found in the present study for men and women [29].

In Latin American countries, a collaborative work that described malnutrition in all its forms made it possible to understand the panorama of some middle-income countries. Overweight and obesity were the main problems of malnutrition in childhood and women. When observing the prevalence of overweight or obesity, as in our study, adult women with low schooling were 17 points more prevalent when compared to normal weight women. Therefore, schooling is a protective factor for adult women [35]. 
A review study performed in 2015 drawing on global data reported similar findings to those of the present study. A negative correlation between social class and family income with obesity was found. Women had consistently higher rates of overweight and obesity, the lower their income. The authors hypothesized that low-paid work for men generally involved greater physical effort and, therefore, men on lower incomes have higher energy expenditure than women in the same situation. Education also showed a strong negative association with overweight/obesity, especially in women [36].

Income played a transforming role regarding the risk of obesity last century. In the mid-twentieth century, wealth correlated with obesity in the USA and Europe, i.e., the greater one's wealth, the higher the likelihood of having excess weight. In the last few decades, however, perhaps due to the abundance and wide availability of unhealthy foods in high-income countries, together with shifts in sociocultural norms, this relationship has changed. Currently, wealth in the USA tends to be negatively correlated with obesity, where individuals classified as poor, or below the poverty line, appear to have higher prevalence rates of obesity. Interestingly, in the present study, this pattern was not evident in males. Greater education showed a positive association with weight change in men. The factors underlying sex differences for this association remain unclear. Although the variables social class or income were not explored in the present study, a positive relationship exists between education and income, where individuals with more years of formal education are more likely to have a higher income [34]. The mechanisms by which income can impact health include: greater access to higher quality material resources, such as food and housing, better access to services that can improve health directly (such as health services and leisure activities), or indirectly, such as education and promotion of self-esteem, and socioeconomic position [37].

The variable area of residence showed a significant association only for men. Men living in rural areas tended to gain less weight than their urban counterparts. This pattern might be due to differing characteristics of foods, with greater consumption of ultraprocessed foods in urban areas [38], or due to other aspects, such as frequency of occupational physical activity in the rural setting [39].

Evidence shows that, globally, there has been a steeper rise in BMI in rural areas than in urban areas. Between 1985 and 2017, the mean increase in BMI was $2.09 \mathrm{~kg} / \mathrm{m}^{2}(95 \%$ CI $1.73 ; 2.44)$ and $2.10 \mathrm{~kg} / \mathrm{m}^{2}(95 \%$ CI $1.79 ; 2.41)$ in women and men from rural areas, respectively, versus $1.35 \mathrm{~kg} / \mathrm{m}^{2}(95 \%$ CI $1.05 ; 1.65)$ and $1.59 \mathrm{~kg} / \mathrm{m}^{2}(95 \%$ CI $1.33 ; 1.84)$ in women and men living in urban settings [40]. In general, there has been a pattern of rising obesity rates in rural areas over recent years, a shift attributed to the modernization of society. Modernization has led to improvements in work tools and instruments, as well as to mechanization and automation in rural work, resulting in a reduction of work-related physical activity among rural dwellers [39]. However, the increase in weight, on average, is still greater in urban areas, suggesting that physical inactivity and/or poor diet are more common in this population. Despite the gap in the literature on the level of physical activity in individuals from rural and urban areas, studies show that the diet of rural dwellers is generally healthier than that of urban dwellers. According to a Brazilian study, individuals residing in rural areas are more likely to have a traditional diet that includes fresh or minimally-processed foods, particularly beans, together with a lower intake of ultra-processed foods, despite lower consumption of fruit and vegetables and fish [38].

The present study has some limitations. This was a cross-sectional study in which respondents assessed for events had different ages at the time of interview. Weight at 20 years of age was reported retrospectively and thus was susceptible to memory recall bias. Individuals from the older age groups may present a cumulative effect for this systematic error, leading to under or over-estimating of the weight reported. The number of indigenous and Asian individuals in the sample was low, hampering meaningful interpretation of differences relative to the other racial groups. Overall, however, the sample size and consistency and coherence with the literature support the validity of the findings of the present study. 
The main strengths of the study are the national representativeness of the sample of 20,000 individuals, a wide diversity of the study population in terms of socioeconomic and demographic aspects, as well as the ability to stratify the respondents by sex. Lastly, the study was based on individual weight and BMI information for different time points, allowing the tracking of changes in nutritional status into adulthood. Therefore, the study results can add to the knowledge in the field by bridging the gap in the literature on weight gain in adulthood, an area which few Brazilian studies have explored to date [23-25,41].

\title{
5. Conclusions
}

The progressive weight gain seen over time contributed to the occurrence of obesity, representing a major public health issue, given the magnitude of the problem and negative health consequences. A higher weight gain was found among the younger group, loweducated women, and high-educated men from urban areas. These results point to the need to strengthen public policies that recognize the social determinants of health and that foster and promote the adoption of healthy lifestyles. Moreover, these results underscore the importance of caring for individuals with overweight and obesity, given that excess weight can lead to the development or exacerbation of other NCDs.

\begin{abstract}
Author Contributions: Conceptualization, N.A.B.S. and D.S.C.; methodology, N.A.B.S. and D.S.C.; software, D.S.C.; formal analysis, N.A.B.S.; investigation, N.A.B.S., K.A.R.-D., J.C.C. and D.S.C.; resources, D.S.C.; data curation, N.A.B.S., K.A.R.-D., J.C.C. and D.S.C.; writing-original draft preparation, N.A.B.S.; writing-review and editing, K.A.R.-D., J.C.C. and D.S.C.; supervision, D.S.C.; funding acquisition, D.S.C. All authors have read and agreed to the published version of the manuscript.
\end{abstract}

Funding: This study was financed in part by the Coordenação de Aperfeiçoamento de Pessoal de Nível Superior-Brasil (CAPES)-Finance Code 001, and had the support of the Fundação de Amparo à Pesquisa do Estado do Rio de Janeiro (FAPERJ) (process numbers E-26/202.667/2018 and E26/010.002203/2019). CAPES and FAPERJ did not have any role in the design of the study and collection, analysis, and interpretation of data and in writing the manuscript.

Institutional Review Board Statement: The National Health Survey was approved by the National Commission on Research Ethics for Humans (Conep) of the National Board of Health (CNS), under permit $n^{\circ} 328.159$. The information contained in the database is confidential since specific data about each household such as identification of the household members, address and telephone is not publicly available.

Informed Consent Statement: Informed consent was obtained from all subjects involved in the study.

Data Availability Statement: The data that support the findings of this study are openly available in the Brazilian Institute of Geography and Statistics (IBGE) at https://www.ibge.gov.br/ estatisticas / sociais / saude/9160-pesquisa-nacional-de-saude.html?=\&t=microdados (accessed on 20 February 2022).

Conflicts of Interest: The authors declare no conflict of interest.

\section{References}

1. WHO. Obesity: Preventing and Managing the Global Epidemic; Technical Report Series; World Health Organization: Geneva, Switzerland, 2000; No. 894; p. 252.

2. Risk Factor Collaboration. Worldwide trends in body-mass index, underweight, overweight, and obesity from 1975 to 2016 : A pooled analysis of 2416 population-based measurement studies in 128.9 million children, adolescents, and adults. Lancet 2017, 390, 2627-2642. [CrossRef]

3. Dai, H.; Alsalhe, T.A.; Chalghaf, N.; Riccò, M.; Bragazzi, N.L.; Wu, J. The global burden of disease attributable to high body mass index in 195 countries and territories, 1990-2017: An analysis of the Global Burden of Disease Study. PLoS Med. 2020, 17, 1084. [CrossRef] [PubMed]

4. Brasil; Ministério da Saúde; Secretaria de Vigilância em Saúde; Departamento de Vigilância de Doenças e Agravos não Transmissíveis e Promoção da Saúde. Vigitel Brasil 2006: Vigilância de Fatores de Risco e Proteção para doenças Crônicas por Inquérito Telefônico; Ministério da Saúde: Brasília, Brazil, 2007. Available online: https://bvsms.saude.gov.br/bvs/publicacoes/vigitel_ brasil_2006.pdf (accessed on 27 February 2021). 
5. Brasil; Ministério da Saúde; Secretaria de vigilância em saúde; Departamento de Vigilância de Doenças e Agravos não Transmissíveis e Promoção da Saúde. Vigitel Brasil 2016: Vigilância de Fatores de Risco e Proteção para doenças Crônicas por Inquérito; Ministério da Saúde: Brasília, Brazil, 2017; 160p. Available online: https://bvsms.saude.gov.br/bvs/publicacoes/vigitel_brasil_ 2016_fatores_risco.pdf (accessed on 6 February 2020).

6. Brasil; Ministério da Saúde; Secretaria de vigilância em saúde; Departamento de Vigilância de Doenças e Agravos não Transmissíveis e Promoção da Saúde. Vigitel Brasil 2019: Vigilância de Fatores de Risco e Proteção para doenças Crônicas por Inquérito; Ministério da Saúde: Brasília, Brazil, 2020. Available online: http://bvsms.saude.gov.br/bvs/publicacoes/vigitel_brasil_2019 _vigilancia_fatores_risco.pdf (accessed on 10 March 2021).

7. Kapoor, E.; Faubion, S.S.; Kling, J.M. Obesity Update in Women. J. Women Health 2019, 28, 1601-1605. [CrossRef] [PubMed]

8. Chagas, D.C.; das Silva, A.A.M.; da Ribeiro, C.C.C.; Batista, R.F.L.; Alves, M.T.S.S.d.B.e. Efeitos do ganho de peso gestacional e do aleitamento materno na retenção de peso pós-parto em mulheres da coorte BRISA. Cad. Saude Publica 2017, 12, e00007916. [CrossRef]

9. Gallon, C.W.; Wender, M.C.O. Estado nutricional e qualidade de vida da mulher climatérica. Rev. Bras. Ginecol. Obs. 2012, 34, 175-183. [CrossRef]

10. Pinto, K.A.; Griep, R.H.; Rotenberg, L.; Conceic, M. Gender, time use and overweight and obesity in adults: Results of the Brazilian Longitudinal Study of Adult Health (ELSA-Brasil). PLOS ONE 2018, 13, e0194190.

11. Canella, D.S.; Levy, R.B.; Martins, A.P.B.; Claro, R.; Moubarac, J.-C.; Baraldi, L.; Cannon, G.; Monteiro, C.A. Ultra-Processed Food Products and Obesity in Brazilian Households (2008-2009). Votruba SB, editor. PLoS ONE 2014, 9, e92752. [CrossRef] [PubMed]

12. Monteiro, C.A.; Cannon, G.; Levy, R.B.; Moubarac, J.C.; Louzada, M.L.C.; Rauber, F.; Khandpur, N.; Cediel, G.; Neri, D.; Martinez-Steele, E.; et al. Ultra-processed foods: What they are and how to identify them. Public Health Nutr. 2019, 22, 936-941. [CrossRef]

13. Askari, M.; Heshmati, J.; Shahinfar, H.; Tripathi, N.; Daneshzad, E. Ultra-processed food and the risk of overweight and obesity: A systematic review and meta-analysis of observational studies. Int. J. Obes. 2020, 44, 2080-2091. [CrossRef]

14. Dietz, W.H. Global shifts in the patterns of urban and rural weight increase. Nat. Med. 2019, 25, 1035-1036. [CrossRef]

15. Monteiro, C.A.; Cannon, G.; Lawrence, M.; Laura Da Costa Louzada, M.; Machado, P.P. Ultra-Processed Foods, Diet Quality, and Health Using the NOVA Classification System. 2019. Available online: http://www.wipo.int/amc/en/mediation/rules (accessed on 27 February 2021).

16. Damiani, D.; Damiani, D. Obesidade monogênica: Um contínuo aprendizado. Pediatrics 2011, 33, 35-44.

17. IBGE. Pesquisa Nacional de Saúde 2013; IBGE: Rio de Janeiro, Brazil, 2014.

18. Word Health Organization. Physical Status: The Use and Interpretation of Anthropometry. Report of a WHO Expert Committee. Am. J. Hum. Biol. 1996, 8, 786-787. [CrossRef]

19. Brasil; Ministério da Saúde; Secretaria de vigilância em saúde; Departamento de Vigilância de Doenças e Agravos não Transmissíveis e Promoção da Saúde. Vigite Brasil 2018: Vigilância de Fatores de Risco e Proteção para doenças Crônicas por Inquérito; Ministério da Saúde: Brasília, Brazil, 2019; 162p. Available online: http://bvsms.saude.gov.br/bvs/publicacoes/vigitel_brasil_2018 (accessed on 7 February 2020).

20. Stopa, S.R.; Szwarcwald, C.L.; Oliveira, M.M.; de Gouvea, E.d.C.D.P.; Vieira, M.L.F.P.; de Freitas, M.P.S.; Sardinha, M.V.; Macário, E.M. Pesquisa Nacional de Saúde 2019: Histórico, métodos e perspectivas. Epidemiol. Serv. Saude Rev. Sist. Unico Saude Bras. 2020, 29, e2020315. [CrossRef] [PubMed]

21. Zheng, Y.; Manson, J.E.; Yuan, C.; Liang, M.H.; Grodstein, F.; Stampfer, M.J.; Willett, W.C.; Hu, F.B. Associations ofweight gain from early to middle adulthood with major health outcomes later in life. JAMA 2017, 318, 255-269. [CrossRef] [PubMed]

22. Jaacks, L.M.; Vandevijvere, S.; Pan, A.; McGowan, C.J.; Wallace, C.; Imamura, F.; Mozaffarian, D.; Swinburn, B.; Ezzati, M. The obesity transition: Stages of the global epidemic. Lancet Diabetes Endocrinol. 2019, 7, 231-240. [CrossRef]

23. Peixoto, M.d.R.G.; Cordeiro, M.d.M.; Ferreira, V.R.; Cardoso, C.K.d.S.; Crispim, P.A.A. Ganho de peso na vida adulta: Preditor da hipertensão arterial? Cad. Saúde Coletiva 2017, 25, 58-64. [CrossRef]

24. Coelho, M.S.P.H.; de Assis, M.A.A.; Moura, E.C. Aumento do índice de massa corporal após os 20 anos de idade e associação com indicadores de risco ou de proteção para doenças crônicas não transmissíveis. Arq. Bras. Endocrinol. Metabol. 2009, 53, 1146-1156. [CrossRef]

25. Brebal, K.M.; Silveira, J.A.; Menezes, R.C.; Epifânio, S.B.; Marinho, P.D.; Longo-Silva, G. Weight gain and changes in nutritional status of brazilian adults after 20 years of age: A time-trend analysis (2006-2012). Rev. Bras. Epidemiol. 2020, 23 , e200045. [CrossRef] [PubMed]

26. Koebnick, C.; Smith, N.; Huang, K.; Martinez, M.P.; Clancy, H.A.; Kushi, L.H. The prevalence of obesity and obesity-related health conditions in a large, multiethnic cohort of young adults in California. Ann. Epidemiol. 2012, 22, 609-616. [CrossRef]

27. Allman-Farinelli, M.A.; Chey, T.; Bauman, A.E.; Gill, T.; James, W.P.T. Age, period and birth cohort effects on prevalence of overweight and obesity in Australian adults from 1990 to 2000. Eur. J. Clin. Nutr. 2008, 62, 898-907. [CrossRef]

28. Berti, T.L.; Rocha TF da Curioni, C.C.; Verly Junior, E.; Bezerra, F.F.; Canella, D.S.; Faerstein, E. Consumo alimentar segundo o grau de processamento e características sociodemográficas: Estudo Pró-Saúde. Rev. Bras. Epidemiol. 2019, 22, e190046. [CrossRef] [PubMed]

29. Canella, D.S.; Duran, A.C.; Claro, R.M. Malnutrition in all its forms and social inequalities in Brazil. Public Health Nutr. 2020, 23, s29-s38. [CrossRef] [PubMed] 
30. Oraka, C.S.; Faustino, D.M.; Oliveira, E.; Teixeira, J.A.M.; de Souza, A.S.P.; Luiz, O.d.C. Raça e obesidade na população feminina negra: Uma revisão de escopo. Saúde Soc. 2020, 29, 1-10. [CrossRef]

31. Paradies, Y. A systematic review of empirical research on self-reported racism and health. Int. J. Epidemiol. 2006, 35, 888-901. [CrossRef]

32. Do Sacramento, A.N.; do Nascimento, E.R. Racismo e saúde: Representações sociais de mulheres e profissionais sobre o quesito cor/raça. Rev. Esc. Enferm. USP 2011, 45, 1142-1149. [CrossRef]

33. Gee, G.C.; Ro, A.; Gavin, A.; Takeuchi, D.T. Disentangling the Effects of Racial and Weight Discrimination on Body Mass Index and Obesity Among Asian Americans. Am. J. Public Health 2008, 98, 493. [CrossRef]

34. Salvato, M.A.; Ferreira, P.C.G.; Duarte, A.J.M.A. The impact of education about income distribution. Estud. Econ. 2010, 40, 753-791. [CrossRef]

35. Batis, C.; Mazariegos, M.; Martorell, R.; Gil, A.; Rivera, J.A. Malnutrition in all its forms by wealth, education and ethnicity in Latin America: Who are more affected? Public Health Nutr. 2020, 23, s1-s12. [CrossRef]

36. Hruby, A.; Hu, F.B. the epidemiology of obesity: A big picture. Pharmacoeconomics 2015, 33, 673-689. [CrossRef]

37. Galobardes, B.; Shaw, M.; Lawlor, D.A.; Lynch, J.W.; Smith, G.D. Indicators of socioeconomic position (part 1). J. Epidemiol. Community Health 2006, 60, 7-12. [CrossRef]

38. De Paula Costa, D.V.; Lopes, M.S.; de Deus Mendonça, R.; Malta, D.C.; de Freitas, P.P.; Lopes, A.C.S. Diferenças no consumo alimentar nas áreas urbanas e rurais do Brasil: Pesquisa Nacional de Saúde. Cien Saude Colet 2021, 26, 3805-3813. [CrossRef]

39. Martins-Silva, T.; dos Santos Vaz, J.; de Mola, C.L.; Assunção, M.C.F.; Tovo-Rodrigues, L. Prevalence of obesity in rural and urban areas in Brazil: National health survey, 2013. Rev. Bras. Epidemiol. 2019, 22, 1-16.

40. Bixby, H.; Bentham, J.; Zhou, B.; Di Cesare, M.; Paciorek, C.J.; Bennett, J.E.; Taddei, C.; Stevens, G.A.; Rodriguez-Martinez, A.; Carrillo-Larco, R.M. Rising rural body-mass index is the main driver of the global obesity epidemic in adults. Nature 2019, $569,260-264$.

41. Conde, W.L.; Borges, C. O risco de incidência e persistência da obesidade entre adultos brasileiros segundo seu estado nutricional ao final da adolescência. Rev. Bras. Epidemiol. 2011, 14, 71-79. [CrossRef] 